\title{
Multi-Grey Image of Laser Marking Gravure Cylinder Based on optimized halftoning Method
}

\author{
Zifen $\mathrm{He}^{1, \mathrm{a}}$, Yinhui Zhang ${ }^{1, \mathrm{~b}^{*}}$ \\ ${ }^{1}$ Kunming University of Science and Technology,Faculty of mechanical and electrical engineering, \\ Kunming, 650500, China \\ azyhhzf1998@163.com, byinhui_z@163.com
}

Keywords: Optimized Halftoning; Multi-Grey Image; Quality Evaluation; Laser Marking

\begin{abstract}
The laser marking intaglio plates were printed in intaglio proofing machine. Three halftoning algorithms for multi-grey image were proposed. The printing and scanning proofs were compared with the simulation images of digital halftoning after scanning the proofs. The quality of images was evaluated by halftoning image quality evaluation method based on human visual system. The experiment results showed that the "bright spots" on the spectrogram gradually tend to be evenly distributed and the error decreases more with increasing clustering partition. Halftone noise level can be aptly described by the proposed method. The residual noise change and uniform characteristics of the smooth region were given for the halftoning image edge and detail place. The results provide effective basis for objective description of halftone image quality.
\end{abstract}

\section{Introduction}

The application of lasers for print plate fabircation plays an increasingly important role in pre-printing area. Laser engraving carving style is a kind of imitation print output device scanning engraving method. Laser engraving gravure output means is controlled by laser switch state ,high energy laser beam irradiating on the surface of the work-piece in the specific point leads the point to rapidly generate evaporation,forming a concave point on the surface of the work-piece. So for the gray scale image, the output must be passed before the digital halftone technology for laser output converted to black and white two value image. Removing the laser wavelength, pulse frequency, quality of laser beam and processing properties of materials and other factors. Gray scale image halftone algorithm for laser image output effect plays a crucial role. Halftoning is a method for creating the illusion of continuous tone output with a binary device. According to computational style, the halftoning algorithms can be classified into three categories [1]: neighborhood based methods, such as error diffusion [2]. Point based methods, such as screening [3] and Look-Up-Table[4]. In general, the halftone images generated by the screening method are visually worse than those generated by the error diffusion method. Iterative optimization methods[5] use a human visual system model to minimize the perceived error.

\section{The Halftone Technology}

\section{Region Analysis}

K-means is one of the simplest unsupervised learning algorithms that solve the well known clustering problem[6]. The main idea is to define $\mathrm{k}$ centroids, one for each cluster. This algorithm aims at minimizing an objective function, in this case a squared error function. The objective function

$$
J=\sum_{j=1}^{k} \sum_{i=1}^{n}\left\|x_{i}^{(j)}-c_{j}\right\|^{2}
$$

where $\left\|x_{i}^{(j)}-c_{j}\right\|^{2}$ is a chosen distance measure between a data point $x_{i}^{(j)}$ and the cluster center $c_{j}$, is an indicator of the distance of the $\mathrm{n}$ data points from their respective cluster centers. 


\section{Edge Detail Analysis}

Suppose we are given a gray-scale image $\left[x_{i, j}\right]$, where $x_{i, j}$ denotes the pixel located at the $i$-th column and the $j$-th row of a grid. Thus the gray-scale image array $\left[x_{i, j}\right]$ and the binary image array $\left[b_{i, j}\right]$ have the same dimensions. We are also given a printer model with the sliding-window form[7]. In the proposed approach, we seek the halftone image that minimizes the weighted squared error

$$
E_{\lambda}=\sum_{k=1}^{4} \lambda_{k} \sum_{i, j}\left(z_{i, j}-w_{i, j}\right)^{2}
$$

where $z_{i, j}=n\left(x_{i, j}\right) * h_{i, j}^{\prime} \quad, \quad w_{i, j}=n\left(p_{i, j}\right) * h_{i, j}$ and $p_{i, j}=P\left(W_{i, j}\right)$. The $\lambda_{k}(\mathrm{k}=1,2,3,4)$ are weighting operators and $W_{i, j}$ is composed of $b_{i, j}$ and *indicates convolution. Note that we have allowed different impulse responses $h_{i, j}, h_{i, j}^{\prime}$ for the eye filters corresponding to the halftone and continuous-tone images.

Multi-scale Error Measure Analysis

For an input represented by a list of $2^{n}$ numbers, the Haar wavelet transform may be considered to simply pair up input values, storing the difference and passing the sum. This process is repeated recursively, pairing up the sums to provide the next scale, finally resulting in $2^{n}-1$ differences and one final sum.

Since most of the wavelet coefficients have small values while only a few wavelet coefficients have large values. Upon the assumption that the wavelet coefficients are mutually independent, they are normalized.The boundary information of the image target is fused by the wavelet coefficients of the correlation between wavelet transform layer, which to increase the pixel resolution scale. We apply the inter-scale fusion method to gain fusion coefficient of the fine-scale, which take into account the detail of the image and approximate information.

In the proposed approach, we seek the halftone image that minimizes the weighted squared error

$$
E_{i, j}=\left\{\sum_{l=1}^{4}(L H, H L, H H)+\sum_{k=1}^{4} \lambda_{k}\right\} \sum_{i, j}\left(z_{i, j}-w_{i, j}\right)^{2}
$$

The $\lambda_{k}(k=1,2,3,4)$ are weighting operators and $*$ indicates convolution. Note that we have allowed different impulse responses for the eye filters corresponding to the halftone and continuous-tone images.

\section{Experimental Materials and Methods}

Object of study is the axial length of $1.3 \mathrm{~m}$ and $0.8 \mathrm{~m}$ gravure printing cylinder, which was engraved by use of German SCHEPERS's laser engraving machine. First, the gravure cylinder general corrosion of copper and its surface to form a traditional ink hole whose depth of about $50 \mu \mathrm{m}$, while determining the grid angle and overprint logo. Next, using electrostatic spraying of the epoxy resin coating formulation in particular gravure cylinder surface, followed by heat treatment and grinding. Thermosetting epoxy and copper-based performance is similar to the surface what can be smoothed by grinding. Mainly to do more to prepare for laser engraving, ready to roll can be stored for a long time, engraving plate for use at any time.

When engraving, a laser beam of high power bursts the drum surface and the epoxy resin vaporized. Adjusting the degree of focus of the laser beam, which enables to reach the depth of the ink cells and size desired. Through multiple bursts, making the final hole carved out of ink smooth and clear. During engraving, the drum to $10 \mathrm{r} / \mathrm{min}$ at a speed of rotation of the bed, the laser beam to $75 \mathrm{~mm} /$ min speed of the drum lateral surface fire. Engraving speed of 100,000 inking cells / s. Intaglio engraving plates and network points shown in Fig.1. 


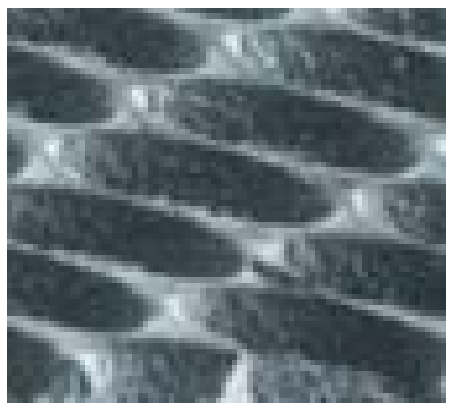

(a)

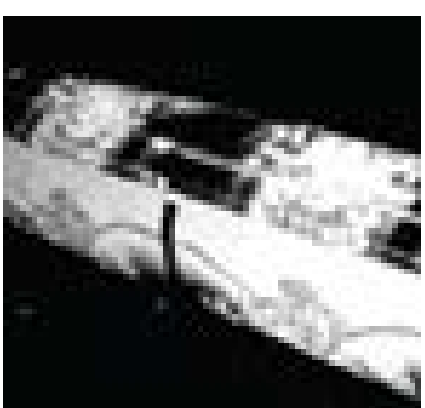

(b)

Fig.1 Gravure cell shape and plate

(a)cell shape (b) gravure plate

\section{Experimental results and discussions}

Halftone image is a special kind of binary images, which multiple gradation reproduction dependents on the dot area and the image "is not the overall smooth", but still showed a black and white image which can not show images "edges and details." . This is a halftone image of spatial characteristics.Frequency domain image reflects dramatic changes in the spatial extent of gray, which is the rate of change in the gray image. The edge portion of the image is mutated portion, rapid changes, so that the reaction in the frequency domain is a high frequency component. The image noise is high frequency portion. That is, the Fourier transform provides the other point of view to observe the image, the image may be converted from the distribution to the frequency distribution of gradation up observation image characteristics.

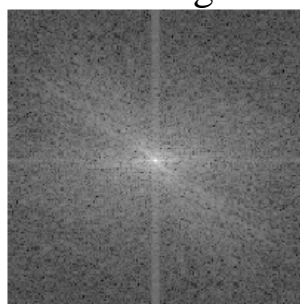

(a)

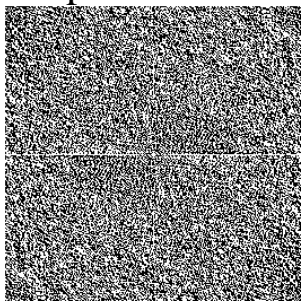

(b)

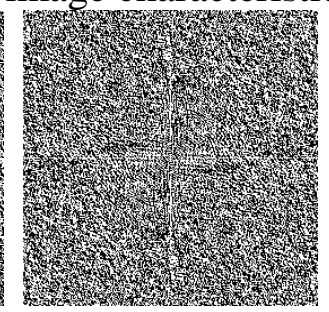

(c)

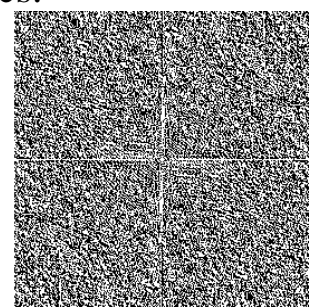

(d)

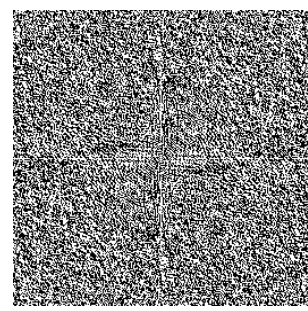

(e)

Fig.2 Frequence spectrum using the discrete Fourier transform (a) Original image frequence spectrum figure (b)Lsmb halftoning frequence spectrum image(c) Proposed algorithm in $\mathrm{K}=2$ with weighed operator $\lambda_{1}, \lambda_{2}$ frequence spectrum image(d)Proposed algorithm in $\mathrm{K}=3$ with weighed operator $\lambda_{1}, \lambda_{2}$ and $\lambda_{3}$ frequence spectrum image (e) Proposed algorithm in $K=4$ with weighed operator $\lambda_{1}, \lambda_{2}, \lambda_{3}$ and $\lambda_{4}$ frequence spectrum image

The corresponding frequency of the image is two-dimensional, which are mapped to the case of the pixel values of the image in two mutually perpendicular directions change. Spectrum shifted after the Fourier transform of the image obtained tends to two "bright line" that will appear on the vertical and horizontal directions. Comparing two types of spectrum halftone image can be seen with increasing cluster partition on the spectrum "bright spot" gradually tend to be distributed evenly, reducing errors more, gray value distribution and smooth transition.

The error between $x_{i, j}$ and $b_{i, j}$ is calculated in the spatial domain where is $e_{i, j}=x_{i, j}-b_{i, j}$, whose results are residual plots of original and halftone figure in two-dimensional discrete Fourier transform. To analyze and understand each frequency domain component of the image performance, this result with the original image of the Fourier spectrum calculation error: $E_{u, v}=F_{u, v}-B_{u, v}$, where $F_{u, v}=\operatorname{DFT}\left(x_{i, j}\right), \quad B_{u, v}=\operatorname{DFT}\left(b_{i, j}\right)$. The resulting $E_{u, v}$ is the halftoning noise spectrum. The resulting spectrum is shown in Fig. 3. 


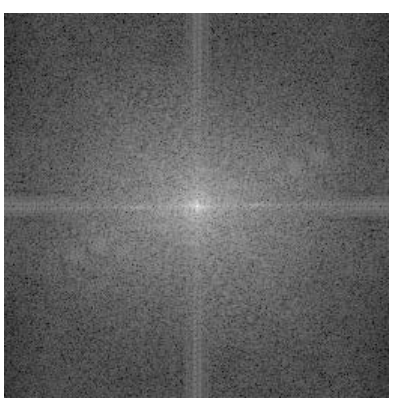

(a)

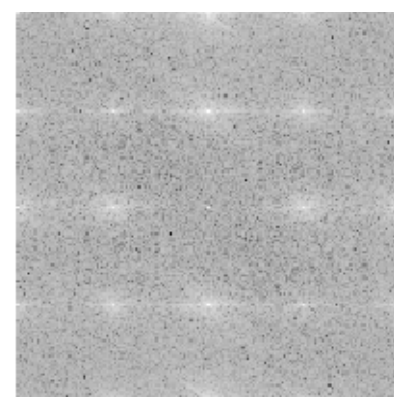

(b)

Fig.3 The original and halftoning spectrum image (a)The original spectrum image(b) halftoning noise spectrum

Compared with the original spectrum, all of Eu,v noise spectrum distributed over a certain frequency components in the corresponding area, the lower the spectral components of the original image. The halftoning noise spectrum is distributed only in the high frequency band and without a strong periodic component. The partial spectral increase from low to high and not sensitive to human vision.

\section{Summary}

The experimental image frequency domain is analyzed and the results showed that with increasing cluster partition on the spectrum "bright spot" gradually moving to distribution, the proposed human visual effects halftone algorithm is better than the model based on least squares algorithm gray value distribution and smooth transition. By analyzing the halftone noise spectrum found halftone noise spectrum is only distributed in the high frequency band and without a strong periodic component. The partial spectral components gradually increase from low to high, which is not sensitive to human vision.

\section{Acknowledgments}

This work was supported by Project 61302173 and 61461022 of the National Science Foundation of China. Kunming university of science and technology talent training fund(14118777)

\section{References}

[1] J.P. Allebach, Ed. vol. MS 154. SPIE:Bellingham, WA(1999).

[2]R. W. Floyd and L. Steinberg.in Proc. Soc. Inf. Display, 1975.p.36

[3]B.E. Bayer. IEEE 1973 International Con\$ on Communications, Vol.26.p.11

[4]P. Li and J. Allebach. In Proc. 1998 IEEE Int. Conf. Image Processing, Chicago, IL, Oct. 1998.p.559

[5]M. Analoui and J. Allebach. in Proc. 1992 SPIE/IS\&T Symp. Electronic Imaging Science Technology, vol. 1666, San Jose, CA, Feb. 1992.p.96

[6]S. N. Sulaiman,N. A. Mat Isa. IEEE Transactions on Consumer Electronics,2010,p.2661

[7]T.N. Pappas,D. L. Neuhoff. IEEE Trans. Image Processing,1999,8(8),p.1102 